\title{
Metastasi linfonodali e recidiva del microcarcinoma papillare tiroideo
}

\author{
Fabio Lanfranco $\cdot$ Stefano Allasia
}

Pubblicato online: 19 novembre 2014

(c) Springer International Publishing AG 2014

\section{Commento a:}

Nodal metastasis and recurrence in papillary thyroid microcarcinoma.

A. Pisanu, A. Saba, M. Podda, I. Reccia, A. Uccheddu. Endocrine (2014) doi:10.1007/s12020-014-0350-7

Il microcarcinoma papillare della tiroide (PTMC) viene definito come un tumore di diametro $\leq 1 \mathrm{~cm}$. Nonostante la maggior parte dei pazienti con PTMC presenti un decorso clinico favorevole e un'eccellente prognosi, in alcuni casi la neoplasia può esordire con l'interessamento dei linfonodi locoregionali e può comportarsi in maniera più aggressiva.

Questo studio retrospettivo ha indagato quali elementi anamnestici e caratteristiche istologiche del tumore tiroideo primitivo aumentino il rischio di metastasi linfonodali alla diagnosi e quali fattori prognostici influenzino la comparsa di recidiva linfonodale nel PTCM.

Sono stati considerati 556 casi di carcinoma della tiroide differenziato diagnosticati tra il 1998 e il 2013, di cui 449 $(80,8 \%)$ carcinomi di tipo papillare. Tra questi, 219 pazienti presentavano una diagnosi istopatologica di microcarcinoma papillare in accordo con la classificazione WHO del 2004. In questa coorte, oggetto dello studio, 24 pazienti mostravano metastasi ai linfonodi cervicali (N1), mentre 195 non presentavano alla diagnosi interessamento linfonodale macroscopico (N0).

La diagnosi citologica preoperatoria di tumore primitivo della tiroide è risultata significativamente più frequente nel gruppo N1 rispetto al gruppo N0. La dimensione media del tumore era maggiore nei pazienti con metastasi linfonodali

F. Lanfranco $(\varangle) \cdot S$. Allasia

Divisione di Endocrinologia, Diabetologia e Metabolismo, Dipartimento di Scienze Mediche, Università di Torino, Torino, Italia

e-mail: fabio.lanfranco@unito.it
(7,5 vs $5,7 \mathrm{~mm}$ ). Nessun paziente con tumore di diametro $<3 \mathrm{~mm}$ presentava metastasi linfonodali alla diagnosi, mentre una neoplasia di diametro $>8 \mathrm{~mm}$ era più frequentemente diagnosticata nel gruppo N1 (45,8 vs 23,0\%). La presenza della variante classica di carcinoma papillare, di tumore multicentrico, di neoplasia con estensione extra-tiroidea (T3) e una diagnosi non incidentale risultavano significativamente più frequenti nel gruppo $\mathrm{N} 1$ rispetto al gruppo N0.

Nel gruppo N1 la tiroidectomia totale è stata associata a dissezione dei linfonodi del compartimento centrale in 4 pazienti, dei linfonodi del compartimento centrale/laterocervicale monolaterale in 14 pazienti e di quelli del compartimento centrale/laterocervicale bilaterale in 6 pazienti.

Durante il periodo di follow-up, 5 pazienti (4 del gruppo N1) hanno presentato recidiva linfonodale, diagnosticata in tutti i casi mediante FNAC.

In conclusione, questo studio ha messo in evidenza la presenza di due tipologie di PTMC, differenziate in base a specifiche caratteristiche anatomopatologiche identificate dopo l'intervento chirurgico. Un tumore multifocale, di diametro $>8 \mathrm{~mm}$, e la presenza di invasione dei tessuti molli peri-tiroidei costituiscono fattori di rischio indipendenti per interessamento linfonodale alla diagnosi. L'invasione dei tessuti extra-tiroidei rappresenta invece il fattore prognostico sfavorevole principale per recidiva linfonodale nei pazienti che alla diagnosi si presentano con metastasi linfonodali. Gli autori dello studio sottolineano quindi l'importanza di utilizzare, di fronte a casi di PTMC aggressivi, un protocollo di trattamento basato sulla stratificazione del rischio e sullo stadio del tumore, che prevede l'uso della terapia radiometabolica e della somministrazione di l-tiroxina a dosi soppressive in associazione all'intervento chirurgico radicale, del tutto analogo a quello utilizzato per la cura del carcinoma papillare della tiroide convenzionale. 